\title{
Reform and practice of optical coherence tomography (OCT) system-driven teaching for optoelectronic instrument principle and design
}

Zhifang Li, Youwu He, Hui Li

Zhifang $\mathrm{Li}$, Youwu He, Hui Li, "Reform and practice of optical coherence tomography (OCT) system-driven teaching for optoelectronic instrument principle and design," Proc. SPIE 10452, 14th Conference on Education and Training in Optics and Photonics: ETOP 2017, 1045260 (16 August 2017); doi: $10.1117 / 12.2269901$

Event: 14th Conference on Education and Training in Optics and Photonics, ETOP 2017, 2017, Hangzhou, China 


\title{
Reform and practice of optical coherence tomography (OCT) system-driven teaching for optoelectronic instrument principle and design
}

\author{
Zhifang Li, Youwu He, Hui Li*
}

College of Photonic and Electronic Engineering, Fujian Normal University, Fuzhou, Fujian 350007, China

*hli@fjnu.edu.cn

\begin{abstract}
Optoelectronic instrument principle and design includes the optical, mechanical, electrical and count modules for one system. We change traditional mode of customary specialty course design for only taking the cell design ability into account. Optical coherence tomography (OCT) can provide high-resolution 3D imaging system and wide application for tissue in vivo. In this work, we carry out OCT system- driven teaching into execution in the course design teaching, and decompose OCT system into four modules for teaching progress. The reform is not only cultivating student design ability based on OCT system exploitation, improving the engineering ability, but also help scientific research promote teaching process.
\end{abstract}

Keywords: optical coherence tomography, system- driven teaching, optoelectronic instrument principle and design

\section{INTRODUCTION}

Optoelectronic instrument principle and design mainly includes the optical, mechanical, electrical and count units for one system. However, these four modules are completely independently in the traditional teaching mode. There is no connection among the units and lack of system and integrity. Teaching contents in each unit are isolated and far from the actual projects, which resulted in the poor design ability and the week general application ability and even the bade whole concept. The reason is that the contents and teaching method of the course design is based on the curriculum, not the course system.

In order to overcome the problem, we choose the advanced optoelectronic instrument-optical coherence tomography (OCT) as the object for teaching contents. We carry out OCT system-driven teaching into execution in the course system teaching, and decompose OCT system into four modules for teaching progress. The teaching method guarantees all contents including integrated system design, and helps foster students' capacity of system design.

OCT is a high-resolution, cross-sectional, three-dimensional imaging modality [1-8]. We have studied OCT system and its application for over ten years [9-10], and have accumulated the enough knowledge for teaching.

14th Conference on Education and Training in Optics and Photonics: ETOP 2017, edited by Xu Liu,

Xi-Cheng Zhang, Proc. of SPIE Vol. 10452, 1045260 · (C) 2017 ICO, IEEE, OSA, SPIE

CCC code: $0277-786 \mathrm{X} / 17 / \$ 18 \cdot$ doi: $10.1117 / 12.2269901$ 


\section{IDEAS OF TEACHING REFORM}

OCT is based on the principle of white light Michelson interferometer. OCT can be classified into time domain OCT(TD-OCT) and Fourier domain OCT (FD-OCT). Their mainly difference is design of reference arm. Reference arm is moving in the TD-OCT, but static in the FD-OCT. FD OCT had two forms: spectral domain OCT (SD-OCT) and swept source OCT (SS-OCT). For teaching reform, we mainly employed SD-OCT, and then brief introduce the knowledge about TD-OCT and SS-OCT. Figure 1 show the schematic diagram of the proposed SD-OCT system. According to SD-OCT system, the teaching contents should contain the choice of optical light source, optical elements and detector, the choice of optomechanical components, DAQ choice and signal sampling control, and image reconstruction and image process shown in Figure 2.

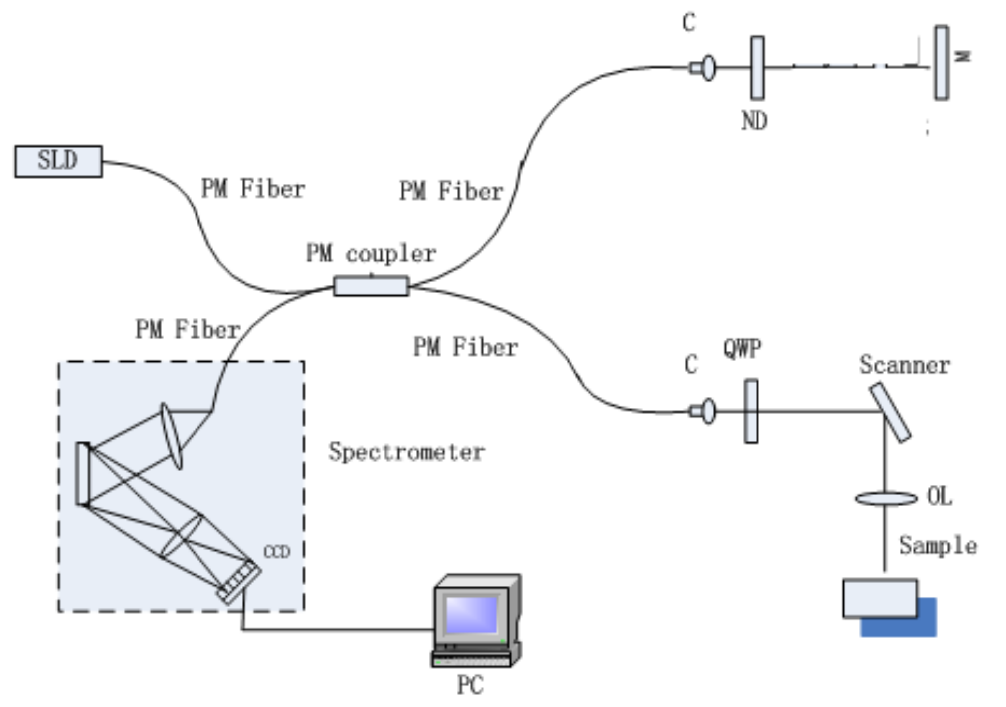

Figure 1. Schematic of the SD-PSOCT system, SLD: superluminescent diode. PM Fiber: polarization maintaining fiber. C: collimator. ND: neutral density filter. BS: non polarization beam splitter. QWP: quarter-wave plate. OL: objective lens. M: mirror

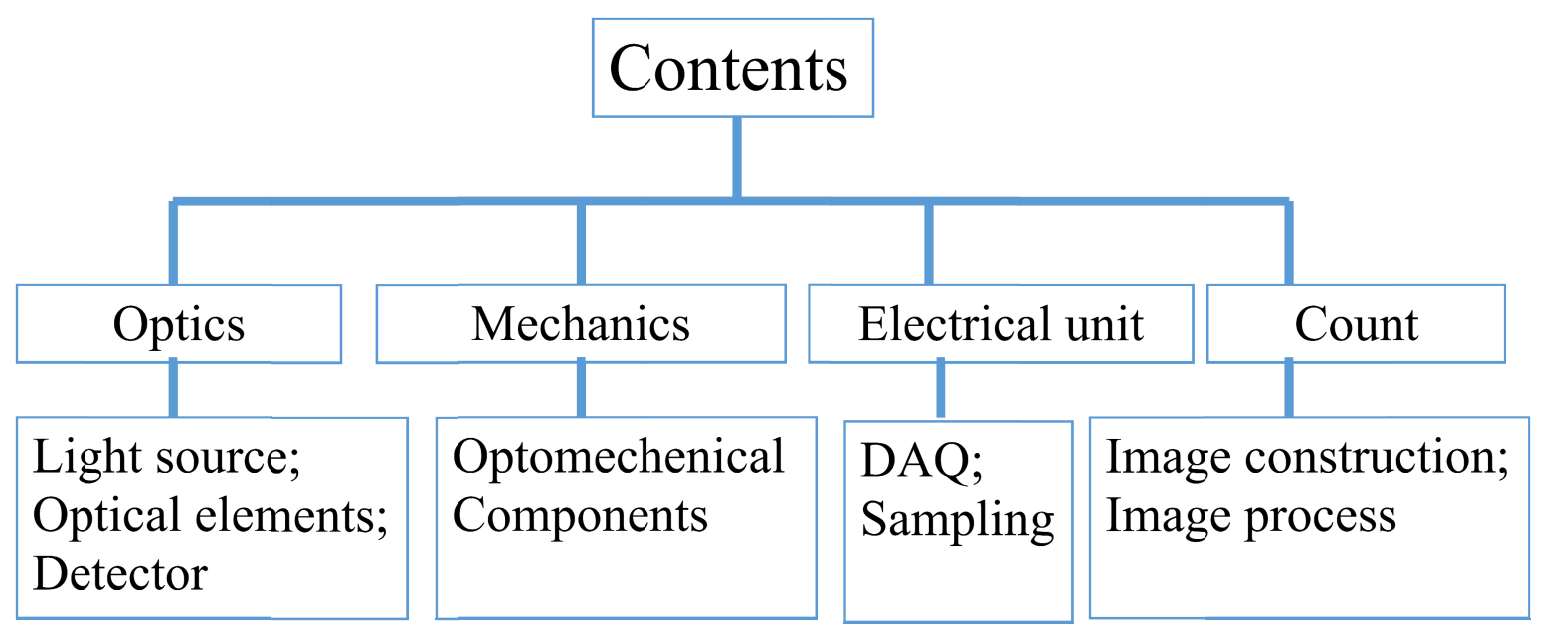

Figure 2 Teaching contents based on SD-OCT system 


\section{SOLUTION OF TEACHING REFORM}

Optical module is how to select the optical source, optical elements and detector. In order to produce the high axial resolution, the light source of wide wavelength bandwidth should be considered as shown in Eq.(1). The parameters of light source was a superluminescent diode (SLD, S5FC1021S, Thorlabs In.) with a FWHM bandwidth of $85 \mathrm{~nm}$ centered at $1310 \mathrm{~nm}$. Choice of light source is the beginning of optoelectronic instrument design.

$$
\Delta z=l_{c}=\frac{2 \ln 2}{\pi} \frac{\lambda^{2}}{\Delta \lambda} \approx 0.44 \frac{\lambda^{2}}{\Delta \lambda}
$$

where $\lambda$ is the central wavelength, and $\Delta \lambda$ is the wavelength bandwidth.

After introduction of SLD for OCT system, the principle, wavelength distribution and application of different light sources (such as lamp, light emitting diodes, SLD, and laser shown in Figure 3) are instructed.

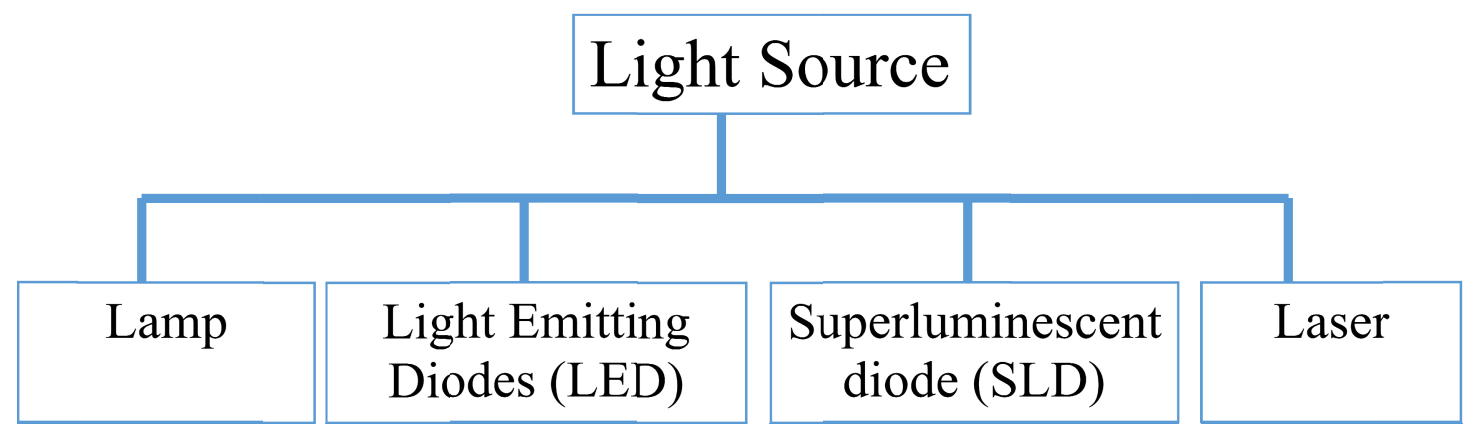

Figure 3. Type of light source

The optical elements containing fiber coupler, mirrors, scanner, neutral density filter, objective lens, and collimator. were chosen based on wavelength range of light source. In the Figure 1, the light coupled to a $2 \times 2$ fiber coupler (TW1300R5F2, Thorlabs In.) and then to be splits into the reference (50\%) and sample (50\%) arms. In the reference arm, neutral density filter was placed in the beam path to enhance the image contrast. In the sample arm, the light was delivered onto the sample by an XY galvanometer scanner (GVS212, Thorlabs In.) and an achromatic lens with a focal length of $50 \mathrm{~mm}$. In order to improve the lateral resolution [shown in Eq. (2)], the $10 \times$ objective lens (LSM02, Thorlabs, Inc.; effective focal length $18 \mathrm{~mm}$, working distance $7.5 \mathrm{~mm}$ ) was employed in sample arm [10]. The detection arm consists of a spectrometer (Wasatch In.) with a single-line-scan CCD camera, which contains 2048 pixels.

$$
\Delta x=\frac{4 \lambda}{\pi} \frac{f_{o}}{d_{o}}=1.27 \frac{\lambda}{N A}
$$

After introduction of choosing optical elements for OCT system, the principle, material and application of different optical element (such as fiber/GRIN lens, lens/mirror, prism/ filter, grating and polarization-dependent components shown in Figure 4) are instructed. 


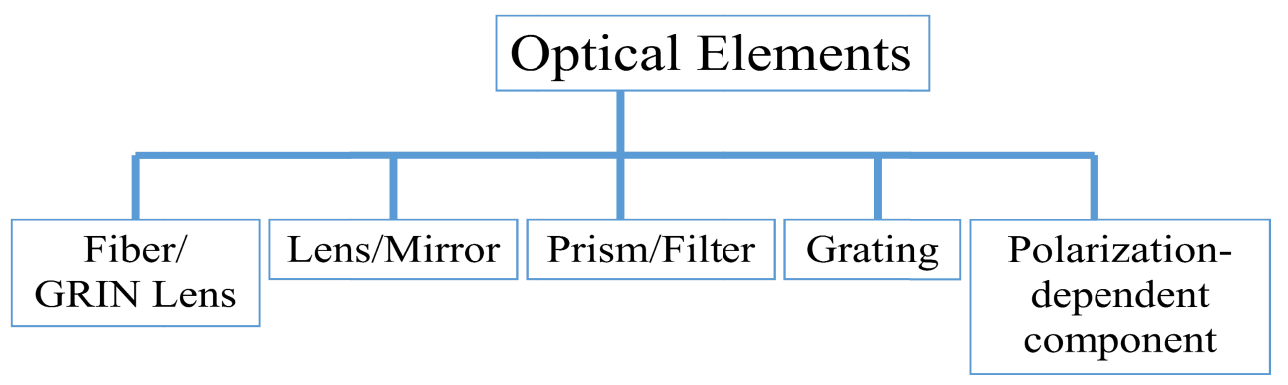

Figure 4. Optical elements

In the three types of OCT, the detectors could be classified into point detector, line-scan CCD and array CCD. Figure 5 show that the teaching contents of detectors included the principle, material, structure, feature and application of three different detectors.

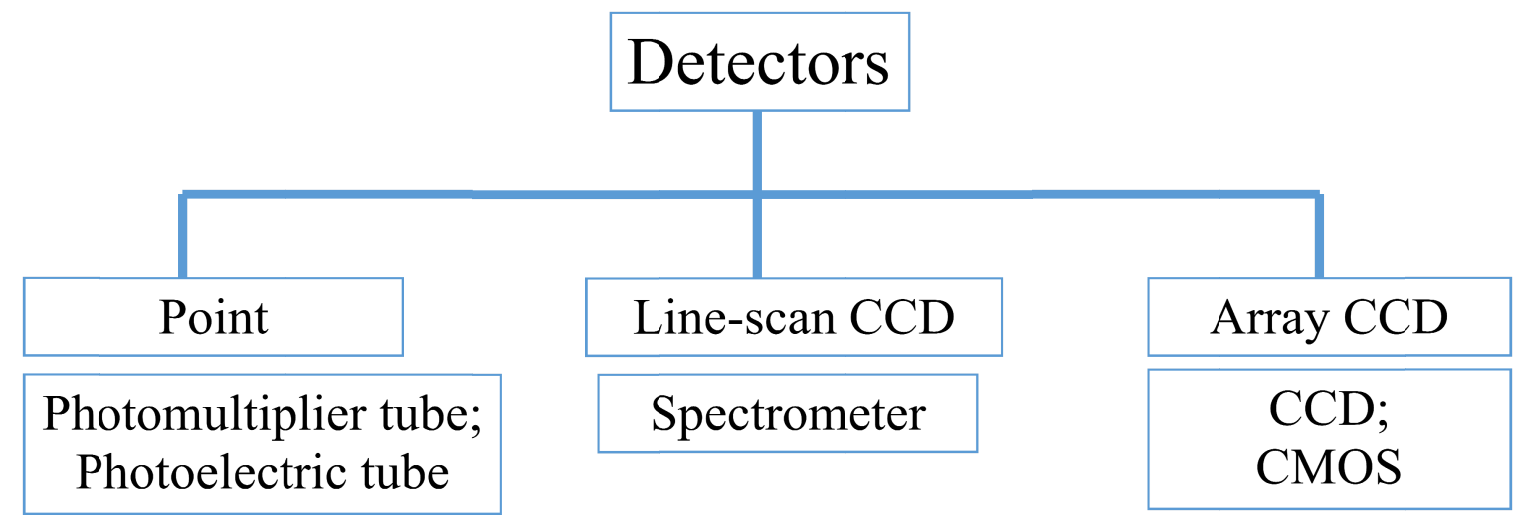

Figure 5. The types of detectors

Mechanical module is how to choose the optomechanical components. The cage system were employed in OCT system. It used four rigid steel rods on which optical components can be mounted along a common optical axis. In addition, the cage system can be integrated into optical setups using mounting posts and/or lens tubes.

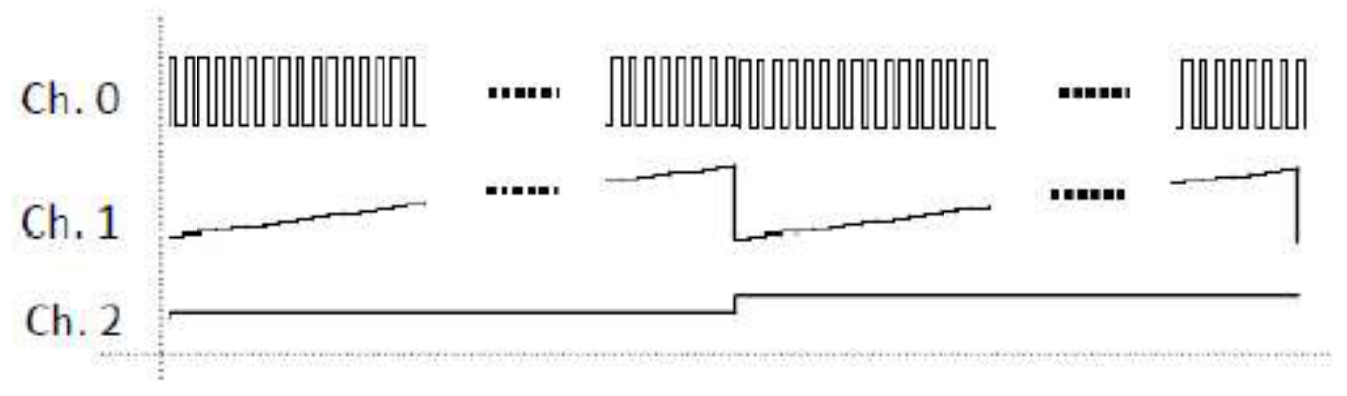

Figure 6. Signal waveforms for system synchronization. Ch. 0: camera trigger signal; Ch.1: B-scan signal; Ch.2: C-scan signal

Electrical module includes the DAQ and sampling control. The signals series which control the scanner (including 
XY galvanometer) and CCD are illustrated in Figure 6. They were generated from DAQ card. A digital pulse train (Ch.0) was generated to trigger the CCD line by line, camera integration time was set at $40 \mu \mathrm{s}$, so the image acquisition speed was $25 \mathrm{k} \mathrm{A}$-line/s. Two voltage signals (Ch.1 and Ch.2) were used to drive the $\mathrm{x}$ - and y-axis galvanometer scanners to acquire the $\mathrm{B}$ - and $\mathrm{C}$-scans respectively.

Count module mainly contains image reconstruction and image process. Image reconstructions of TD-OCT and FD-OCT are different. The reconstruction algorithms of TD-OCT and FD-OCT are Eq. (3) and Eq. (4), respectively. Figure 7 show the main step of image process. There are two parts in the contents. One is the theoretical introduction, the other is the computer experiments.

$$
\begin{aligned}
I_{d}\left(z_{r}\right) & =\frac{\eta}{4}\left[S_{0}\left(R_{r}+R_{s 1}+R_{s 2}+\cdots+R_{s N}\right)\right] \\
& +\frac{\eta}{4}\left[S_{0} \sum_{i=1}^{N} \sqrt{R_{r} R_{s i}} \exp \left[-\left(z_{r}-z_{s i}\right)^{2} \Delta k^{2}\right] \cos \left[2 k_{0}\left(z_{r}-z_{s i}\right)\right]\right]
\end{aligned}
$$

where the first term is the direct current, and the second term is interference components, $S_{0}=\int_{0}^{\infty} S(k) d k$ is the power spectral density function of light source in the k-space.

$$
\begin{aligned}
i_{d}(z)= & \frac{\eta}{8}\left[\gamma(z)\left(R_{r}+R_{s 1}+R_{s 2}+\cdots+R_{s N}\right)\right] \\
& +\frac{\eta}{4} \sum_{i=1}^{N} \sqrt{R_{r} R_{s i}}\left[\gamma\left[2\left(z_{r}-z_{s i}\right)\right]+\gamma\left[-2\left(z_{r}-z_{s i}\right)\right]\right] \\
& +\frac{\eta}{8} \sum_{i \neq j=1}^{N} \sqrt{R_{s i} R_{s j}}\left[\gamma\left[2\left(z_{s i}-z_{s j}\right)\right]+\gamma\left[-2\left(z_{s i}-z_{s j}\right)\right]\right]
\end{aligned}
$$

where the first term is the direct current, the second is the cross-correlation term, and the third is the auto-correlation term. And $r_{s}\left(z_{s}\right)$ is the reflection function at the different layer of sample.

\section{D OCT data}

Pre-precess

Figure 7. Image Process

\section{Features}

In addition, the optical elements, mechanical components, and DAQ and scanning control are the same for three types of OCT.

\section{ACHIEVEMENT OF TEACHING REFORM}

This work connected four modules in the teaching content of optoelectronic instrument principle and design based on 
OCT system to fuse the knowledge system of the students and improve the comprehensive application ability. The teaching contents of four module (optical, mechanical, electrical and count modules) were determined by the OCT system requirements. The students not only strengthen and master relevant theoretical knowledge, but also enhance the capacity of system design through the study of OCT four modules. OCT system-driven teaching help teachers guide students to complete a system design from part to whole, and promote scientific research into teaching process to expand the students' vision. Furthermore, students complete a project and improve the students' comprehensive design ability.

\section{ACKNOWLEDGEMENTS}

This project was sponsored in part by National Natural Science Foundation of China (No. 81571726), Fujian Provincial Natural Science Foundation (2015J01006).

\section{REFERENCES}

[1] Oykuafutim.C.A.,Hee M.R., Lin C.P., Reichel E., Schuman J.S., Duker J.S., Izatt J.A., Swanson E.A., Fujimoto J.G., "Imaging of macular diseases with optical coherence tomography," Ophthalmology 102, 217-229 (1997)

[2] Chen Z., Milner T.E., Srinivas S., Wang X., Malekafzali A.,van Gemert M.J.C, Nelson J. S., "Noninvasive imaging of in vivo blood flow velocity using optical Doppler tomography," Opt. Lett. 22, 1119-1121 (1997)

[3] Chen Y., Andrews P.M., Aguirre A. D., Schmitt J.M., Fujimoto J.G., "High-resolution three-dimensional optical coherence tomography imaging of kidney microanatomy ex vivo," J. Biomed. Opt. 12(3), 034008 (2007)

[4] Andrews P.M., Chen Y., Onozato M.L., Huang S.-W., Adler D.C., Huber R.A., Jiang J., Barry S.E., Cable A.E., Fujimoto J.G., "High-resolution optical coherence tomography imaging of the living kidney," Laboratory Investigation 88, 441-449 (2008)

[5] Wierwille J., Andrews P.M., Onozato M.L., Jiang J., Cable A., Chen Y., "In vivo, label-free, three-dimensional quantitative imaging of kidney microcirculation using Doppler optical coherence tomography," Laboratory Investigation 91, 1596-1604 (2011)

[6] Zhi Z., Jung Y., Jia Y., An L., Wang R.K., "Highly sensitive imaging of renal microcirculation in vivo using ultrahigh sensitive optical microangiography," Biomed. Opt. Express, 1059-1068 (2011)

[7] Li Q., Onozato M. L., Andrews P.M., Chen C.-W., Paek A., Napha R., Yuan S., Jiang J., Cable A., Chen Y., " Automated quantification of microstructural dimensions of the human kidney using optical coherence tomography (OCT)," Opt. Express 17, 16000- 16016 (2009)

[8] Andrews, P.M., Wang H.W., Wierwille J., Gong W., Verbessey J., Cooper M., Chen Y., "Optical coherence tomography of living human kidney," Journal of Innovative Optical Health Science, 7, 1350064 (2014)

[9] Li Z., Li H., He Y., Cai S. and Xie S., " A model of speckle contrast in optical coherence tomography for characterizing the scattering coefficient of homogenous tissues," Phys. Med. Biol. 53, 5859 (2008)

[10] Li Z., Tang Q., Jin L., Andrews P.M., Chen Y., "Monitoring kidney microanatomy changes during ischemia-reperfusion process using texture analysis of OCT images," IEEE Photonics Journal, 9, 4000110 (2017) 